\title{
Particle Tracking Random Simulation of Pollutant Migration and Diffusion
}

\author{
Du Zhehua ${ }^{1, *}$, Lin $\mathrm{Xin}^{2}$ \\ ${ }^{1}$ Wuhan Second Ship Design and Research Institute, 430205 Wuhan, China \\ ${ }^{2}$ Hubei Province Engineering Consulting Co., LTD., 430071 Wuhan, China
}

\begin{abstract}
A two-step Lagrangian particle tracing method was used to simulate the particle movement in convective diffusion. Firstly, the flow field distribution was given by finite difference method. Secondly convective diffusion was simulated by particle tracing method. Jet current through centre slit in the static flow and turbulent flow as well as the side discharge of pollutant were simulated as case study. In this paper, finite difference method is used to solve fluid velocity field distribution, and particle tracking method is used to solve convection-diffusion equation. The result shows that the data produced by particle tracing method is reasonable and the method is stable. This method can be applied to the calculation of the convective diffusion of pollutant and spread to other problems.
\end{abstract}

\section{Introduction}

The current research on the migration and diffusion of pollutants can be roughly divided into two categories. One type is Euler method, which uses numerical calculation and simulation research. Commonly used methods include finite difference method, finite element method and finite volume method. The other is Lagrangian method, also known as particle tracing method. Particle tracing method is seldom used to solve this problem. Particle tracing method can not only be used to simulate randomness problem and diffusion movement of matter particles, but also can be used to solve other problems in fluid.

Particle tracing method has many applications. Li Hai, Ji Shunying et al. [1] comprehensively considered the efficiency of finite difference method in sea ice dynamics calculation under Euler coordinates. They also used smooth particle hydrodynamics (SPH) method in Lagrangian coordinates to accurately simulate the rheological behavior of sea ice. Tang Junjian and $\mathrm{Yu}$ Xingguang et al. [2] established Lagrange water quality point tracking method, which approximately simulated the migration process of suspended sediment in the sea. Kazuyoshi Matsuzaki et al. [3] used Lagrange method to establish particle motion trajectory and calculate it when conducting numerical study on the swirling flow of cyclone dust collector. German scholars Ulf Grawe and Jorg-Olf Wolf [4] used Lagrangian method when studying the ocean dynamics model of suspended particles (SPM). They described convection-diffusion partial differential equations in terms of random motion of particles and show that Lagrange method is more accurate than Euler method. $\mathrm{Li}$ Shuhua and Xia Huayong [5] used particle tracking method to simulate and study the tidal current field and Lagrangian drift of particles in the tipping area of Fangchenggang. Jiang Wensheng and Sun Wenxin [6] simulated the movement of fine particulate matter discharged from Yellow River in Bohai Sea and the transport of suspended particulate matter under strong winds by using the improved threedimensional particle tracking model of suspended particulate matter. Ji Qingfeng et al. [7] established the random model of sediment particle movement with random method, which solved the problem of sedimentation in the front pool of pump. Chen Shairui [7] improved particle tracking method and applied it to the numerical simulation of pollutant diffusion in the Yangtze River Estuary. It can be seen from the above that particle tracking method has a wide range of research applications, and the requirements for research objects and conditions are relatively loose, and the final results are relatively satisfactory. However, there are few reports on the application of this method in solving the problem of convection and diffusion of pollutants. In this paper, particle tracking method in solving the problem of convection and diffusion of pollutant will be analyzed.

\section{Theoretical basis}

\subsection{Particle tracking}

Particle tracing is a method of theoretical mechanics. It regards fluid as a system of particles composed of several particles. Based on the study of the individual fluid particles motion, the motion law of entire fluid is determined by studying the motion of each fluid particle.

\footnotetext{
* Corresponding author: shunli878@163.com
} 
To solve the problem of pollutant migration and diffusion, it is necessary to solve the basic equation of convection and diffusion.

$$
\begin{aligned}
& \frac{\partial C}{\partial t}+u \frac{\partial C}{\partial x}+w \frac{\partial C}{\partial y}=D\left(\frac{\partial C^{2}}{\partial y^{2}}+\frac{\partial C^{2}}{\partial x^{2}}\right) \\
& \frac{\partial C}{\partial t}+u \frac{\partial C}{\partial x}=D \frac{\partial C^{2}}{\partial x^{2}}
\end{aligned}
$$

Equation (1) is a two-dimensional convectiondiffusion equation, and equation (2) is a one-dimensional convection-diffusion equation, which are applicable to different flow field environment. When solving the convection-diffusion equation, the first thing to know is the velocity distribution of each particle in the fluid. Then flow velocity of space point where the particle is located is used to determine the particle flow position in the fluid. Finally, we calculate particle diffusion.

\subsection{Determination of flow position}

Let flow velocity of a mass point in the $x$ direction be $u$. After passing through time $t$, particle is moved from position $Y(0)$ at $t=0$ to position $Y(t)$, which is expressed as the following equation.

$$
Y(t)=Y(0)+\int_{0}^{t} u\left(t^{\prime}\right) d t^{\prime}
$$

Velocity $u$ in the above equation can be solved by using finite difference method to solve velocity equation (4) and (5). The distribution of velocity $u$ (and $w$ ) of spatial nodes is calculated. Then the velocity of particles not on the mesh nodes is interpolated to get velocity $u_{m}$ (and $w_{m}$ ). Finally, the particle movement position $Y(t)$ in the equation is determined by the particle flow velocity.

Equation (4) is differentiated into equation (5).

$$
\begin{aligned}
& \frac{\partial C}{\partial t}+u \frac{\partial C}{\partial x}+w \frac{\partial C}{\partial y}=D\left(\frac{\partial C^{2}}{\partial y^{2}}+\frac{\partial C^{2}}{\partial x^{2}}\right) \\
& \frac{u_{i, j}^{n+1}-u_{i, j}^{n}}{d t}+u \frac{u_{i+1, j}^{n+1}-u_{i, j}^{n+1}}{d x}+w \frac{u_{i, j+1}^{n+1}-u_{i, j}^{n+1}}{d y}= \\
& D_{y} \frac{u_{i, j+1}^{n+1}-2 u_{i, j}^{n+1}+u_{i, j-1}^{n+1}}{d y d y}+D_{x} \frac{u_{i+1, j}^{n+1}-2 u_{i, j}^{n+1}+u_{i-1, j}^{n+1}}{d x d x}
\end{aligned}
$$

\subsection{Basic solution of diffusion equation}

The basic solution of pollution line source diffusion equation $\partial_{t} C=D \nabla^{2} C$ is equations (6) and (7).

$$
\begin{aligned}
& C(x, t)=\frac{M}{\sqrt{2 \pi} \sigma} \exp \left\{-\frac{1}{2} \frac{(x-\mu)^{2}}{\sigma^{2}}\right\} \\
& C(x, y, t)=\frac{M}{2 \pi \sigma^{2}} \exp \left\{-\frac{1}{2} \frac{(x-\mu)^{2}}{\sigma^{2}}-\frac{y^{2}}{\sigma^{2}}\right\}
\end{aligned}
$$

Equations (6) and (7) are the basic solutions of onedimensional and two-dimensional convective diffusion equations, respectively.
In this study, concentration $\mathrm{C}$ is regarded as composed of a large number of particles. Theoretically speaking, density distribution of entire concentration $C(x)$ in the flow field is Gauss normal distribution. From variance $\sigma$ and mean $\mu$, all the characteristics of Gauss concentration distribution can be determined.

For the basic solution of one-dimensional convection-diffusion equation, when $\sigma=1$ and $\mu=0$, the standard form of distribution density is $f(x)=1 / \sqrt{2 \pi} e^{-\frac{1}{2} x^{2}}$.Probability distribution function $F(x)=\int_{-\infty}^{x} f(x) d x$ is a monotonically increasing function from 0 to 1 . In this case, only the distribution of this standard formula is required to obtain the distribution state of concentration $C(x)$.

\section{Typical example}

There are many types of pollutants in water bodies, which can be roughly divided into solubility and subsolubility. First, when simulation object is soluble pollutant, the velocity of pollutant particle is the same as that of fluid, that is, $u_{p}=u$ ( $u_{p}$ is the velocity of pollutant particle, and $u$ is the velocity of flow body). Secondly, when simulation object is insoluble pollutant and particles are small, flow velocity of polluted particles can be approximately equal to fluid flow velocity, that is $u_{p} \approx u$. Finally, when simulation object is insoluble pollutant and particles are large, flow velocity of polluted particles is not equal to the fluid flow velocity, but $\mathrm{u}_{\mathrm{p}}=u \cdot \beta$ ( $\beta$ is velocity coefficient $)$. In the simulation calculation, particle velocity is determined by the above points.

In order to facilitate simulation calculation, in the following three typical calculation examples, this paper will take soluble pollutant as examples, that is, $u_{p}=u$, and put a large number of particles into it for simulation calculation.

\subsection{Pollutant emission problem of center-slit jet in stationary fluid}

There is jet pollution in a stationary fluid. Fluid calculation domain is divided into $30 \mathrm{~m} \times 11 \mathrm{~m}$ grids (Figure 1). Flow velocities $u$ at the initial section jets $(1,5),(1,6)$ and $(1,7)$ is $1 \mathrm{~m} / \mathrm{s}$. The pollutant concentration at the jet is regarded as composed of a large number of particles. The initial sections $(1,5),(1,6)$ and $(1,7)$ have a total of 3000 particles. Each jet point is 1000 mass points.

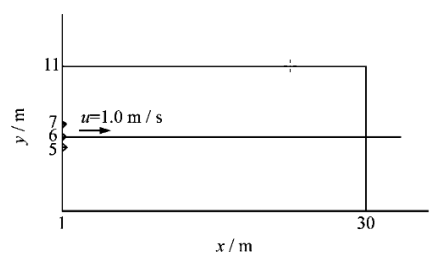

Fig. 1 Schematic diagram of study area

There are 3000 pollutant particles in the initial jet section. These mass points move and spread with the increase of time steps. The diffusion result of 1000 
particles placed at a single jet point (take point $(1,6)$ as an example) at different time steps is shown in Figure 2(a). The migration and diffusion of pollutant particles at other jet points are similar. Because there are also pollutants discharged into fluid at $(1,5)$ and $(1,7)$ on both sides of the ejection point $(1,6)$, they interact with each other and superimpose each other, making the pollutants discharged at the jet flow migrate and diffuse in the fluid. Specific results are shown in Figure 2(b).

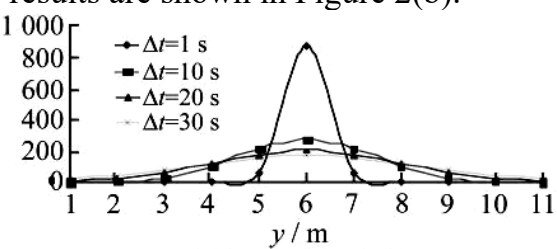

(a)

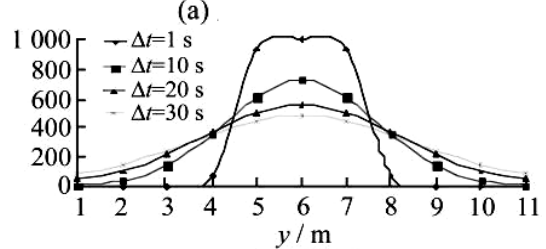

(b)

Fig. 2 Particle diffusion distribution diagram

It can be seen from Figure 2(a) that as time step increases $(n=1,10,20,30$ in $\Delta t \cdot n)$, pollutant points at jet point $(1,6)$ gradually increase from 1,000 to 171 . The total number of pollutant points on each section is 1,000 . Figure 2(b) is the result of diffusion and distribution of fluid polluted particles in the entire study. The total number of pollutant points on each section is 3000 . In order to further prove the rationality of simulation results of particle tracking method, random walk method and particle tracking method are compared under the same calculation conditions. Random walk method can describe complex things with random nature and physical experiment process more realistically, and it is commonly used in random water quality simulation. Figure 3 shows the concentration diffusion curves of different methods at the same time. It can be seen that the overall trend of concentration diffusion distribution simulated by the two methods is similar, and the whole concentration diffusion state conforms to Gauss normal distribution.

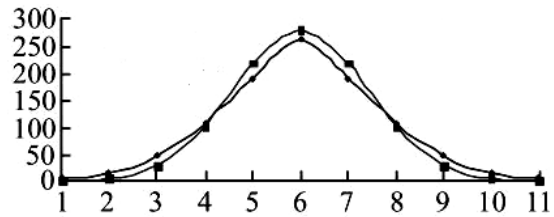

Fig. 3 Comparison of concentration diffusion curves of different methods

\subsection{Jet pollution emission of turbulent fluid}

There is jet pollution in turbulent fluid. The fluid calculation domain is divided into $40 \mathrm{~m} \times 11 \mathrm{~m}$ grids (Figure 4). The pollutant concentration at the jet is regarded as composed of a large number of particles. The initial sections $(1,5),(1,6)$ and $(1,7)$ have a total of 3000 particles. Each jet point has 1000 particles.

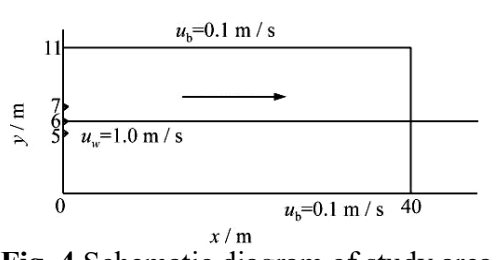

Fig. 4 Schematic diagram of study area

Firstly, exponential distribution formula of velocity of turbulent fluid is used. Secondly, jet velocity distribution calculated above (Fig. 5) is used to calculate the position of jet pollutant particles following the flow. By solving the analytic solution of the diffusion equation (6), the final result is shown in Figure 6 and Figure 7.

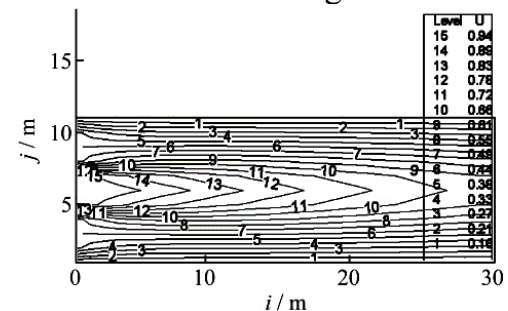

Fig. 5 Jet fluid velocity distribution diagram

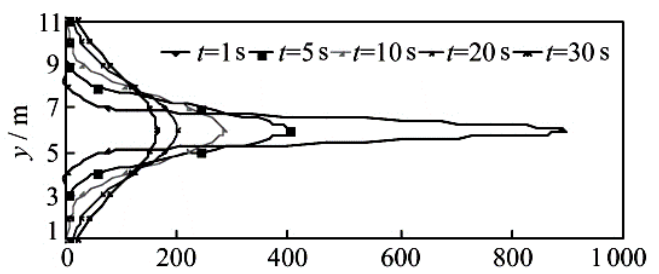

Fig. 6 Concentration distribution diagram of central jet at different moments

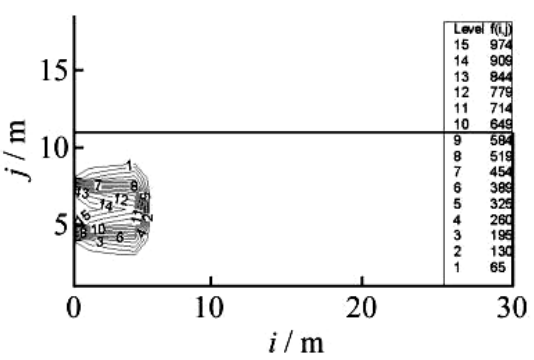

(a) $\Delta t=5 \mathrm{~s}$

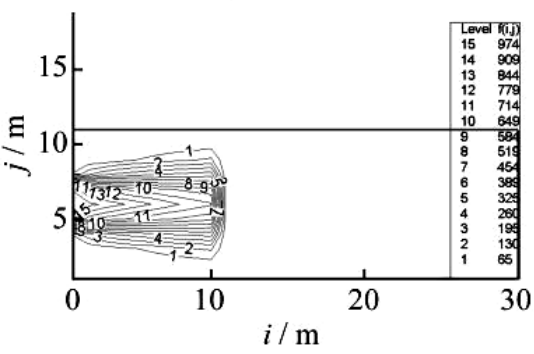

(b) $\Delta t=10 \mathrm{~s}$

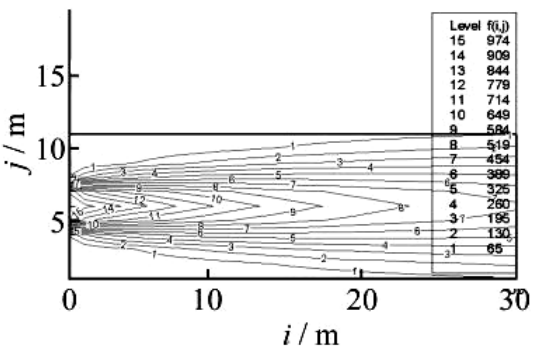

(c) $\Delta t=30 \mathrm{~s}$

Fig. 7 Mass point diffusion result during pollutant discharge 
It can be seen from Figure 5 that the maximum flow velocity at jet exit is $1 \mathrm{~m} / \mathrm{s}$, and the flow velocity gradually decreases along the jet direction. In the flow velocity control range, the minimum flow velocity is about $0.2 \mathrm{~m} / \mathrm{s}$. The flow velocity perpendicular to the direction of jet also gradually decreases, and the minimum flow velocity in the flow velocity control range is about $0.15 \mathrm{~m} / \mathrm{s}$. Figure 6 is the concentration distribution diagram of the central jet of 1000 pollutant particles at different moments. Figure 7 shows the concentration distribution result at different time steps. It is consistent with the overall concentration change trend in comparison with Figure 6. In general, the simulation result is in accordance with the general law of pollution diffusion of slotted jet, and the simulation result is relatively satisfactory.

\subsection{Side blowdown problem}

There is a sewage outlet on the bank of a turbulent fluid to discharge sewage. Fluid calculation domain is divided into $40 \mathrm{~m} \times 11 \mathrm{~m}$ grids (Figure 8 ). In this paper, pollutants discharged from the sewage outlet are regarded as three mass points, which are $m_{1}, m_{2}$, and $m_{3}$ respectively. The migration and dispersion phenomena of $m_{1}, m_{2}$, and $m_{3}$ in fluid is simulated by particle tracing method. The simulation results are shown in Figure 9 and Figure 10.

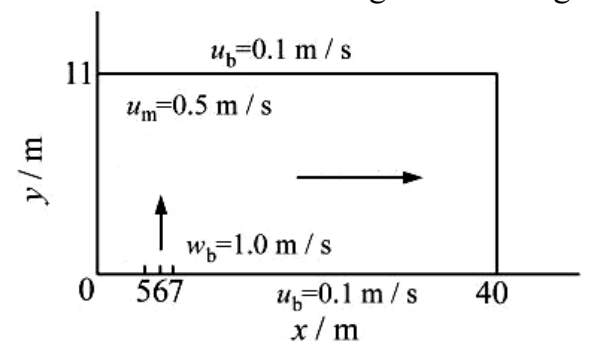

Fig. 8 Velocity boundary condition

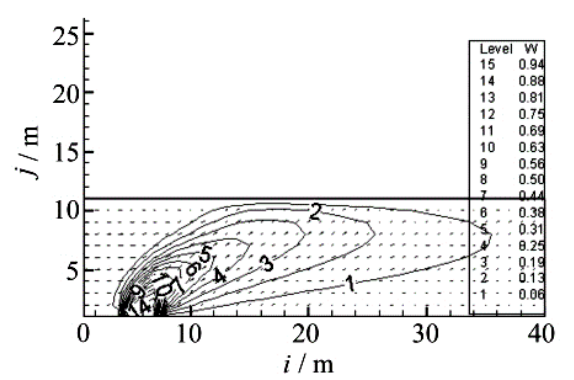

Fig. 9 Velocity profile

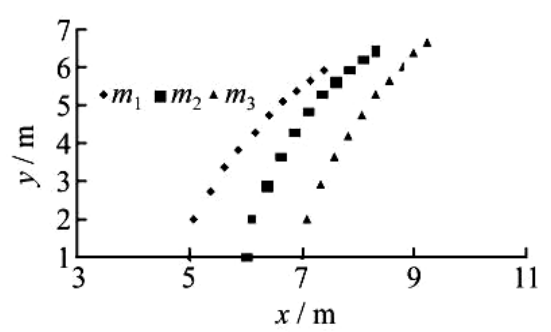

(a) Coordinates of particle $\mathrm{M}_{1}, \mathrm{M}_{2}$ and $\mathrm{M}_{3}$

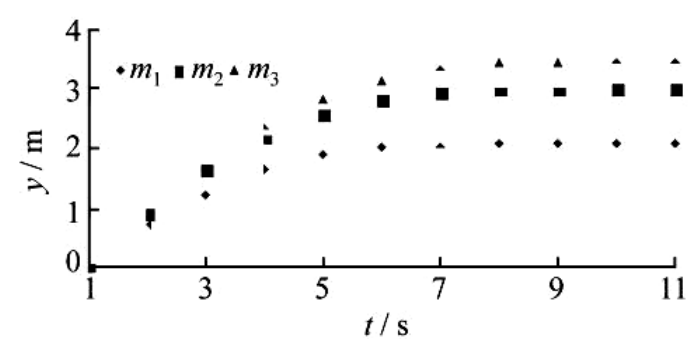

(b) $y$ coordinate of mass point

Fig. 10 Distribution of particle coordinates

Generally speaking, the research mass points become larger with time step. The particles position also change correspondingly, which is consistent with the general trend of diffusion. The diffusion distribution on research mass point also tends to be consistent with Gauss normal distribution, indicating that the result obtained by this method is reasonable.

\section{Conclusion}

Based on the characteristics of particle tracking method, this study proposes a two-step approach to solve the convection and diffusion problem of pollutants, simplifying complex problems and solving practical problems more conveniently and quickly.

Basic equation to solve water pollution problem is convection-diffusion equation. In this paper, finite difference method is used to solve fluid velocity field distribution, and particle tracking method is used to solve convection-diffusion equation. The generated data is more reasonable and stable. This method can be used to solve practical problems.

\section{Acknowledgments}

This work was supported by the National Key Research and Development Program (No.2017YFC0307800) .

\section{References}

1. H. Li, S.Y Ji, H.D. Shen, Acta Oceanologica Sinica, 30, 1 (2008)

2. J.J. Tang, X.G. Yu, J. Chen, Journal of Applied Oceanography, 28, 90 (2009)

3. K. Matsuzaki, H. Ushijima, M. Munekata, Journal of Thermal Science, 15, 181 (2007)

4. U. Grawe, J.O. Wolff, Environmental Fluid Mechanics, 10, 21 (2009)

5. S.H. Li, H.Y. Xia, Guangxi Sciences, 7, 279 (2000)

6. W.S. Jiang, W.X. Sun, Oceanologia et Limnologia Sinica, 32, 94 (2001)

7. Y.F. Qing, Y.M. Chen, L.H. Feng, Journal of Irrigation and Drainage, 23, 40 (2004) 